\title{
Molecular Diversity of miR390-Guided Transacting siRNA Precursor Genes in Lower Land Plants: Experimental Approach and Bioinformatics Analysis
}

\author{
M. S. Krasnikova, ${ }^{1}$ I. A. Milyutina, ${ }^{1}$ V. K. Bobrova, ${ }^{1}$ A. V. Troitsky, ${ }^{1}$ \\ A. G. Solovyev, ${ }^{1}$ and S. Y. Morozov ${ }^{1,2}$ \\ ${ }^{1}$ A.N. Belozersky Institute of Physico-Chemical Biology, Moscow State University, 119992 Moscow, Russia \\ ${ }^{2}$ Department of Virology, Moscow State University, 119992 Moscow, Russia \\ Correspondence should be addressed to S. Y. Morozov, morozov@genebee.msu.su \\ Received 31 July 2011; Revised 28 September 2011; Accepted 28 September 2011 \\ Academic Editor: Alfredo Ciccodicola
}

Copyright ( $) 2011$ M. S. Krasnikova et al. This is an open access article distributed under the Creative Commons Attribution License, which permits unrestricted use, distribution, and reproduction in any medium, provided the original work is properly cited.

\begin{abstract}
Transacting siRNA loci (TAS3-like) of a particular plant species are usually represented by several gene families. PCR-based approach was used as a phylogenetic profiling tool to probe genomic DNA samples from representatives of evolutionary distant Bryophyta taxa, namely, class Bryopsida (subclasses Bryidae and Dicranidae) and class Sphagnopsida. We found relatives of all four Physcomitrella patens (subclass Funariidae) TAS3-like loci in subclasses Bryidae and Dicranidae. Only representatives of subclass Bryidae encoded TAS3-like genes belonging to P. patens TAS3a and TAS3d families. On the other hand, only the members of order Grimmiales (subclass Dicranidae) encoded gene relatives of $P$. patens TAS3c family. These data indicate that moss ta-siRNA families have been long conserved during land plant evolution. However, P. patens TAS3-like loci were detected neither in two Sphagnum species from the earliest diverged moss class Sphagnopsida, nor in the Selaginella kraussiana from the earliest extant tracheophyta lineage, Lycopodiopsida.
\end{abstract}

\section{Introduction}

Plants produce several distinct types of small RNAs that are predominantly 21 to 24 nucleotides in size. Small interfering RNAs (siRNAs) including heterochromatic siRNAs (hcsiRNAs), natural antisense transcript-derived siRNAs (natsiRNAs), trans-acting siRNAs (ta-siRNAs), and secondary siRNAs are involved in transcriptional and posttranscriptional gene silencing [1-10]. Deep sequencing experiments have demonstrated that thousands of loci per genome generate these siRNAs, with $24 \mathrm{nt}$ hc-siRNA loci being predominant $[10,11]$. In contrast, microRNAs (miRNAs) are encoded by perhaps just several hundred loci, which are transcribed with RNA polymerase II (Pol II) to generate singlestranded precursors further processed into specific miRNAs. These precursors contain the mature miRNA sequence within an imperfect double-stranded RNA hairpin, which is processed in the nucleus by Dicer-like (DCL) protein DCL1 to give an imperfect RNA duplex with two-nucleotide $3^{\prime}$-overhangs on each strand. One strand derived from this duplex functions as a miRNA. In total, several dozens of plant miRNA families (hundreds of individual miRNA species) are currently identified and found to target mainly protein-coding mRNAs $[2,4,9,12,13]$.

Endogenous ta-siRNAs arise from Pol II-transcribed genes and function in plants to guide cleavage of target mRNAs [16]. Biogenesis of ta-siRNAs requires RNA-dependent RNA polymerase 6 (RDR6), suppressor of gene silencing 3 (SGS3), and DCL4. Like other siRNAs and miRNAs, tasiRNAs are methylated at $2^{\prime} \mathrm{O}$ of the $3^{\prime}$ terminal nucleotide by HEN1, an RNA methyltransferase. The biogenesis pathway for ta-siRNAs is initiated by a site-specific cleavage of primary TAS transcripts by AGO proteins guided by a miRNA $[7,8,17]$. The processed primary transcript is then converted to a double-stranded (ds)RNA precursor by the activity of RDR6 in cooperation with SGS3. A DCL4 dicer activity then 
catalyzes the precursor cleavage which occurs with 21-nt intervals starting from the end of precursor generated by miRNA-guided cleavage. Active ta-siRNAs, therefore, are accurately phased with respect to the site of miRNA-guided cleavage. The generated 21-nt ta-siRNAs further work as components of an RNA-induced silencing complex (RISC) to guide AGO-dependent cleavage of their target mRNAs. Recent studies have reported that some ta-siRNAs also work as cleavage guiders to cut ta-siRNA precursors transcribed from TAS genes, similarly to miRNA, and thus generate secondary ta-siRNAs with new specificity [17-20]. In Arabidopsis thaliana, eight ta-siRNA-generating loci have been identified that fall into four families (AtTAS1-4). Most TAS gene transcripts have only a single miRNA target site (miR173 for TAS1 and TAS2 and miR828 for TAS4) positioned $5^{\prime}$ to the ta-siRNA-producing region, and the target site is cleaved by AGO1 slicer activity guided by the respective miRNA [21]. However, two miR390 binding sites $\left(5^{\prime}\right.$ and $3^{\prime}$ to the ta-siRNA encoding region) were shown to be necessary for the TAS3 precursor RNA processing, that appeared to be dependent on specific interaction between AGO7 and miR390 [17-20]. In a current model, the biogenesis of AtTAS3-derived ta-siRNAs is initiated by miR390-mediated cleavage of a capped and polyadenylated TAS3 transcript at the $3^{\prime}$ binding site by the AGO7 slicer activity. The miR390AGO7 complex interaction with a noncleavable $5^{\prime}$ binding site appears to be required for recruitment of SGS3 and RDR6 to produce dsRNA spanning the ta-siRNA precursor up to the $5^{\prime}$ binding site for miR390 (M. Pooggin, personal communication). Then DCL4, in cooperation with its binding partner DRB4, processes the resulting dsRNA into 21-nt duplexes starting from the miR390-cleaved terminus [17].

Although ta-siRNA genes (TAS genes) were first found in higher plants [3], their existence also in the moss Physcomitrella patens has been later reported $[22,23]$. The TAS3 family genes and miR390 have been identified in various plant species including eudicots, monocots, and gymnosperm, suggesting a conserved function of the encoded tasiARFs, which play an important role in auxin responses and plant development through posttranscriptional regulation of mRNAs coding for their target transcription factors, auxin response factor 3 (ARF3) and ARF4 [2, 8, 12, 24]. Interestingly, although all four identified P. patens TAS3-like loci code for tasiARF species homologous to those in flowering plants [22] that indicates their extreme evolutionary conservation, no sequenced TAS3-loci were reported in other lower land plants (namely, ferns, lycopods, and bryophytes). However, the data supporting ta-siRNAs formation were obtained for unicellular green algae Chlamydomonas reinhardtii [25]. Moreover, miR390 sequences were revealed in fern (Ceratopteris thalictroides) and moss Polytrichum juniperinum (class Polytrichopsida) that strongly suggests the presence of TAS3-like RNA precursors in these organisms [24].

Previously, we described the new method for identification of plant ta-siRNA precursors based on PCR with oligodeoxyribonucleotide primers mimicking miR390. The method was found to be efficient for dicotyledonous plants, cycads, conifers, and mosses [15]. In this study, PCR-based approach was used as a phylogenetic profiling tool to probe genomic DNA samples derived from Selaginella kraussiana (Lycopodiopsida) and 13 bryophyta species from classes Bryopsida (subclasses Bryidae and Dicranidae) and Sphagnopsida (Sphagnum squarrosum and Sphagnum girgensohnii). We failed to detect TAS3-like loci in Selaginella kraussiana and Sphagnum sp. Nevertheless, we found relatives of all four P. patens TAS3-like loci in Bryidae and Dicranidae species. These results indicate that at least some of ta-siRNA families have been long conserved during land plant evolution.

\section{Materials and Methods}

2.1. Plant Material. Moss specimens for 13 studied species listed in Table 1 and Selaginella kraussiana were taken from collections of the N. V. Cycin Main Botanic Garden of the Russian Academy of Sciences.

2.2. Analysis of Nucleic Acids. Total DNA was isolated from about $200 \mathrm{mg}$ of plant material by Nucleospin Plant Kit (Macherey-Nagel) according to the protocol of the manufacturer. For amplification, the Physcomitrella TAS3-specific primers [15] were used: a forward primer Bryo TAS-P 5' GRCGYTAYCCTCYTGAGCT-3' and reverse primer Bryo TAS-M 5' -TAGCTCAGGAGTGATA(G/T)A(C/A)AA-3' . For PCR, 25-35 cycles were used for amplification with a melting temperature of $95^{\circ} \mathrm{C}$, an annealing temperature of $58^{\circ} \mathrm{C}$, and an extending temperature of $72^{\circ} \mathrm{C}$, each for 30 seconds, followed by a final extension at $72^{\circ} \mathrm{C}$ for $3 \mathrm{~min}$. PCR products were separated by electrophoresis of samples in 1.5\% agarose gel and purified using the GFXTM PCR DNA and Gel Band Purification Kit (Amersham Biosciences). For cloning, the PCR-amplified DNA bands isolated from gel were ligated into pGEM-T (Promega). Cloned products were used as templates in sequencing reactions with the ABI Prism BigDye Terminator Cycle Sequencing Ready Reaction Kit (Applied Biosystems). DNA sequences were deposited at the NCBI data bank, and the accession numbers are shown in Table 1.

2.3. Computational Sequence Analysis. TAS3-like sequences identified in moss species were compared using multiple alignment tool at MAFFT (version 6). The MAFFT package is available at http://mafft.cbrc.jp/alignment/server/. Sequence similarities were additionally analysed by NCBI Blast at http://blast.ncbi.nlm.nih.gov/BlastAlign.cgi. The presence of open reading frames within sequenced amplification products was analysed at http://web.expasy.org/translate/.

\section{Results and Discussion}

Previously, four ta-siRNA loci targeted by miR390 were found in moss Physcomitrella patens and referred to as PpTAS3a-d. All four loci contain $5^{\prime}$ and $3^{\prime}$ miR390-target sites, and ta-siRNAs derived from these moss loci regulate, similarly to those encoded by the angiosperm TAS3 loci, ARF genes and, additionally, target mRNA of AP2 transcription factors [22]. To apply the approach based on PCR amplification [15] to TAS3-like loci of lower land plants, we designed degenerated primers BryoTAS-P and Bryo TAS-M 
TABLE 1: Bryophyta species studied and data on their sequenced TAS3-like loci.

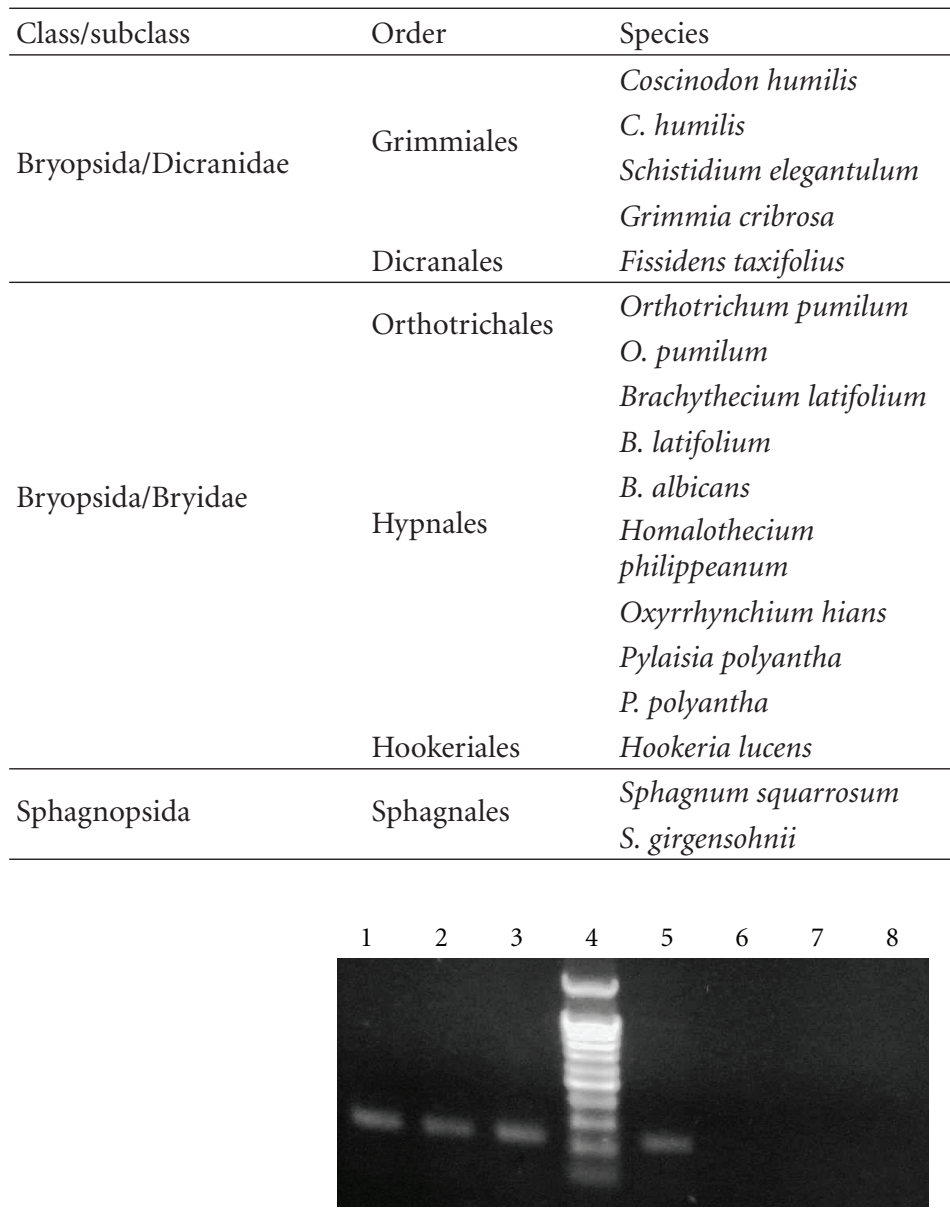

(a)

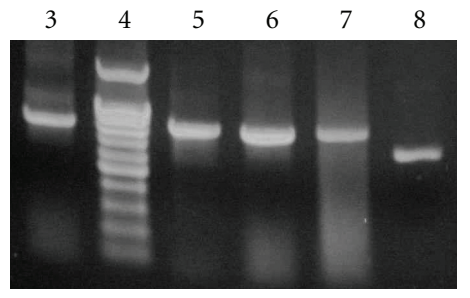

(b)

Figure 1: Analysis of PCR products in 1.5\% agarose gel. Amplification of genomic DNA sequences flanked by miR390 sites. (a) PCR products were obtained on genomic DNAs with moss-specific primers. Orthotrichum pumilum (1), Coscinodon humilis (2), Pylaisia polyantha (3), Homalothecium philippeanum (5), Sphagnum girgensohnii (6), Sphagnum squarrosum (7), and Selaginella kraussiana (8). (4), DNA size markers including bands ranging from $100 \mathrm{bp}$ to $1000 \mathrm{bp}$ with $100 \mathrm{bp}$ step and $1500 \mathrm{bp}$ band (Sibenzyme). (b) PCR products were obtained on genomic DNAs with the internal transcribed spacer-specific (ITS2-specific) primers [14] as a control of genomic DNA preparation quality. Lane numbering corresponds to those in (a).

complementary to miR390-target sites of PpTAS3a-d [22]. These primers were used for PCR on total DNA isolated from mosses belonging to classes Bryopsida and Sphagnopsida as well as from the lycopod Selaginella kraussiana.

PCR amplification of chromosomal DNA from representatives of subclasses Bryidae and Dicranidae resulted in synthesis of major bands of 200-250 bp (Figure 1 and Table 1). Cloning and sequencing of the obtained DNA bands revealed that the amplified sequences similarly to $P$. patens contained well-conserved TAS3 region composed of AP2specific ta-siRNA sequence followed by ARF-specific sequence and located between miR390 target sites corresponding to PCR primers [22]. Moreover, amplified sequences showed obvious similarity to TAS3-like genes from $P$. patens plants (Figures 2 and 3 ) as it was revealed by bioinformatic analysis of the putative TAS3-like sequences using multiple sequence alignment tool MAFFT. NCBI Blast revealed that
Orthotrichum pumilum clone 24-Opu TAS3-like sequence showed $71 \%$ identity to the $P$. patens locus TAS3a genomic sequence, Brachythecium latifolium clone $50-\mathrm{Br}$ sequence showed $72 \%$ identity to the $P$. patens locus TAS3d genomic sequence, Fissidens taxifolius clone 17-Fit sequence showed $76 \%$ identity to the $P$. patens locus TAS3b genomic sequence, and Coscinodon humilis clone 20-Chu showed almost 70\% identity to the $P$. patens locus TAS3c genomic sequence.

As a rule ta-siRNA loci of a particular plant species are represented by several gene families. For example, four tasiRNA loci targeted by miR390 were found in $P$. patens (see above), and three miR390-dependent TAS3 loci were identified in A. thaliana $[12,17]$. It was reasoned that multiple TAS3-like loci might be paralogs derived by duplication of an ancestral locus and subsequent divergence of its copies, and perhaps the areas of TAS3-like loci producing biologically relevant ta-siRNAs may have been preferentially conserved 
$\operatorname{miR} 390$

\begin{abstract}
Physcomitrella patens TAS3d Physcomitrella patens TAS3 Brachythecium latifolium clone $50-\mathrm{Br}$ Orthotrichum pumilum clone $24-\mathrm{Op}$ Pylaisia polyantha clone 11-Pyp
\end{abstract}

Physcomitrella patens TAS3d Physcomitrella patens TAS3 Brachythecium latifolium clone $50-\mathrm{Br}$ Orthotrichum pumilum clone 24-Op Pylaisia polyantha clone 11-Pyp

Phycomitrella patens TaS Physcomitrella patens TAS3d
Physcomitrella patens TAS3a Brachythecium latifolium clone $50-\mathrm{Br}$ Orthotrichum pumilum clone 24-Op Pylaisia polyantha clone 11-Pyp

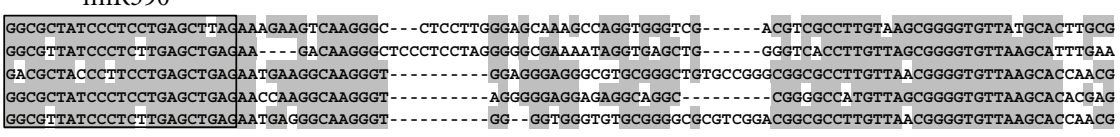

ta-siAP2

ta-siARF

CTTGACGCTCCGATCTAAGACGTCAGCTGTAGCTCCA TGGGGTGTGATGACTGCTTAGCCCGGCACACACTCTCTGCCCGG CCACCTACCCTTGTGAGATGGGTAGCGC TGCAACACTCCTACGCAAGACCCTAGCTATGGCTCCA TAGGGTGTGATGAGTGCTTCA TCCGGTGCTCTTCTACTGCCTTGOCCACCTACCCTTGTGATATGQGCCGCGC GGCGCCCCGGTGGCCTCAGATGTCT GTTATGGCTCC TAGGGTGTGATGAGTGCTTTA CCCAACACTCATCCTCTACGCAGC CCACCTACCCTTGTGACATGGGCGCAGT CCCGGCAGAGCGGCGCCAACCGTGAGGCATGGCCCT TAGGGTGTGATGAGTGCTTTA CACGGCGCTCGTCCTCTGCCCAGOCCACCTACCCTTGTGACATGGGCACCGC $\operatorname{miR} 390$

TGATTTGCGCGACTTCCATGTCG TTTTATATCACTCCTGAGCT GTGTCTGCGTGTCTCCTGTATCGGTTGTATATCACTCCTGAGCT GCTTATCGGCGCAGCCCATGTCA. TTGTATATCACTCCTGAGCT CCTTAGGGGCGCGTCCGTGTCGA TTGTCTATCACTCCTGAGCT2

FIgUre 2: Nucleotide sequence alignment of ta-siARF loci of $P$. patens TAS3a and $\mathrm{d}$ and moss TAS3-like sequences determined in this and previous [15] papers. Shading shows residues identical for the given position in most sequences.

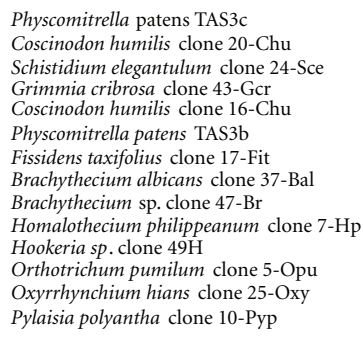

Physcomitrella patens TAS3c Coscinodon humilis clone 20-Chu Schistidium elegantulum clone 24 -Sce Grimmia cribrosa clone 43-Gcr Coscinodon humilis clone 16-Chu Physcomitrella patens TAS3b Fissidens taxifolius clone 17-Fit Brachythecium albicans clone 37-Bal Brachythecium sp. clone 47-Br Homalothecium philippeanum clone 7-Hp Hookeria sp. clone $49 \mathrm{H}$ Orthotrichum pumilum clone 5-Opu Orthotrichum pumilum clone 5-Opu
Oxyrhynchium hians clone 25-Oxy Pylaisia polyantha clone 10-Pyp

Physcomitrella patens TAS3c Coscinodon humilis clone 20-Chu Schistidium elegantulum clone 24 -Sce Grimmia cribrosa clone $43-\mathrm{Gcr}$ Physcomitrella patens TAS3b Physcomitrella patens TAS3b
Fissidens taxifolius clone 17-Fit Fissidens taxifolius clone 17-Fit
Brachythecium albicans clone 37-Bal Brachythecium sp. clone 47-Br Homalothecium philippeanum clone 7-Hp Hookeria sp. clone $49 \mathrm{H}$ Orthotrichum pumilum clone 5-Opu Oxyrrhynchium hians clone 25-Oxy Pylaisia polyantha clone 10-Pyp

Physcomitrella patens TAS3c Coscinodon humilis clone 20-Chu Schistidium elegantulum clone 24-Sce Grimmia cribrosa clone $43-\mathrm{Gcr}$ Coscinodon humilis clone 16-Ch Physcomitrella patens TAS3b Fissidens taxifolius clone 17-Fit Brachythecium albicans clone 37-Bal Brachythecium sp. clone 47-Br Homalothecium philippeanum clone $7-\mathrm{Hp}$ Hookeria sp. clone $49 \mathrm{H}$ Orthotrichum pumilum clone 5-Opu Oxyrrhynchium hians clone 25-Oxy Pylaisia polyantha clone 10-Pyp
$\operatorname{miR} 390$

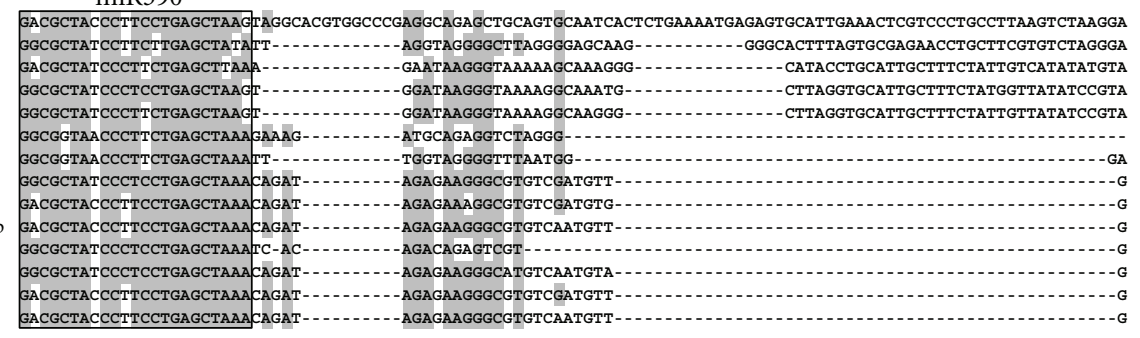

ta-siAP2

ta-siARF

ATAGATTAGG PAGGGTGGAACGATTGCTTAAACA-AGCAAGTTCATATTTGCCCAACCAGCTACCCTTGAGACAAGGCTTGTTTGCCTTTGGGCAAGGGCTTTAGAGATA GTGGAGGGGCTAAGGTGTGATGAGTGCTTTAACA-AACATCTCTCA-CTGGCCCAA CCACGTACACCTGAGACAATGCCCATCTGCCATGGCACAAGGGCTTTTCACCTC GTGGAGGGC PAAGGTGTGATGAGTGCTTTAFCA-AACATCTCTCA-CTGGCCCAA CACGTACACCTGAGACAATGCCCATCTGCCATGGCACAAGGGCTTTTCACCTC
ATACATTGGGPAGGGTGTAGTGAGTGTTTTAECA-AACATATCATC-TTTGCCGCACAAGGTATTCTTGAGACAAAGCCTTCTTTCCATTTCATTTGGGCTTTGCAATTA

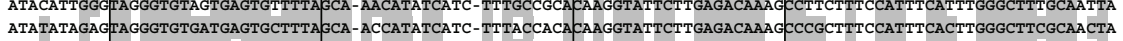
ATATATAGAG AGGGTGTGATGAGTGCTTTAECA-ACCATATCATC-TTTACCACA CAAGGTATTCTTGAGACAAAGCCCGCTTTCCATTTCACTTGGGCTTCGCAACTA
ATATATTGAGPAGGTGTGATGCGTGCTTTAECA-ACCATATCATC- TTTGCCACA CAAGGTATTCTTGAGACAAAGCCCGCTTTCCATTTCACTTGGCTTTGCAATTA ATATATTGAGPAGGGTGTGATGCGTGCTTTAGCA-ACCATATCATC-TTTGCCACA CAAGGTATTCTTGAGACAAAGCCCGCTTTCCATTTCACTTGGGCTTTECAATTA
GAGAACTGGCTAGGGTGTGATGAGTGCTTCCGTA-AGCAACTCATG-CGAGCTCAA.CCACGTACCCTTGAGACAAGACCCGTCTGCCATCGCACAAGGGCAACGCACCAT GAGAACTGGCPAGGGTGTGATGAGTGCTTCCGTA-AGCAACTCATG-CGAGCTCAA CACGTACCCTTGAGACAAGACCCGTCTGCCATCGCACAAGGGCAACGCACCAT
GTGGAGTGGTPAAGGTGTGATGAGTGCTTTTAGA-AGCATCTCTCA-TTAACCCAACCACATACACCTGAGACAGGACCCATCTGCCATGGCACAAGGGCTTTACACCTC GTGGAGTGGT PAAGGTGTGATGAGTGCTTTTAGA-AGCATCTCTCA-TTAACCCAACACATACACCT GAGACAGGACCCATCTGCCATGGCACAAGGGCTTTACACCTC GCGTGGGGGCPAAGGTGTGATGAGTGCTTTAECATGACATCTCTCT-TATGCTCAACCACGCACTCCAGAGACAAGACCCGTCTGTCATGACACAAGGGCTTCGCACCTT GCGTGAGGGC PAAGGTGTGATGAGTGCTTTA_GCATGACATCTCTCT-TATGCTCAA_CACGTACTCCAGAGACAAGACCTGTCTGTCATGACACTAGGGCTTCGCACCTT GCGTGTGGGCPAAGGTGTGATGAGTGCTTTAECAGATCATCTCCCCGCGAGCCCGA CACAGACTGTAGTCACACGGCCCGTCTGTTATGACACAAGGGCTTCGCAGCTT GCGTGAGGGC PAAGGTGTGATGAGTGCTTTAGCATAACATCTCTCA-TATGCGCAA CCACGTACTCCAGAGTCAAGGCCTGTCTGTCATAACTCAAGGGCTTCGCACCTT GCGTGAGGGC PAAGGTGTGATGAGTGCTTTAECATGACATCTCTCT- TATGCTCAA CCACGTACTCCAGAGACGAGACCCATCTGTCATGACACAAGAGCTTCGCACCTT GCGTGAGGGC एAAGGTGTGATGAGTGCTTTAAGCATGACATCTCTCT-TATGCTCAA_CACGTACTCCAGAGACAAGACCTGTCTGTCATGACACTAGGGCTTCGCACCTT

$\operatorname{miR} 390$

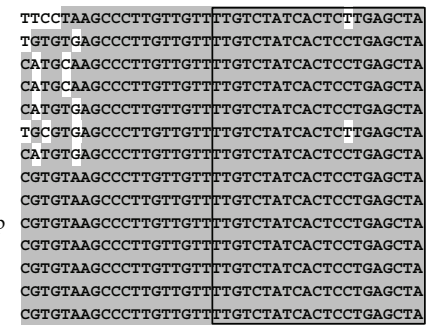

Figure 3: Nucleotide sequence alignment of ta-siARF loci of $P$. patens TAS3b and $\mathrm{c}$ and moss TAS3-like sequences determined in this paper and previous [15] papers. Shading shows residues identical for the given position in most sequences.

$[20,22]$. However, despite obvious progress in phylogenetic studies of miRNA precursor evolution in plants $[2,4,9,12$, 27], little is known about TAS gene molecular evolution. In this paper, we attempted to estimate the diversity of TAS3like genes in moss species from class Bryopsida (subclasses Bryidae and Dicranidae) and subdivide them according to gene families known for $P$. patens - a representative of Funariidae, another subclass of Bryopsida (Figure 4). Sequencing of clones from major amplified DNA bands showed that Brachythecium latifolium, Coscinodon humilis, Orthotrichum pumilum, and Pylaisia polyantha encode at least two types of miR390-dependent AP2/ARF-targeting TAS loci demonstrating sequence similarity to different families of TAS loci characterized for Physcomitrella patens (Figures 2 and 3). Totally, we investigated 11 Bryopsida moss species (Table 1). Six of them belong to orders Hookeriales and Hypnales, which together comprise more than $25 \%$ of all known moss species [14]. Subclass Dicranidae (totally 4 species studied) was mainly represented by plants from order Grimmiales (Figure 4 and Table 1). 


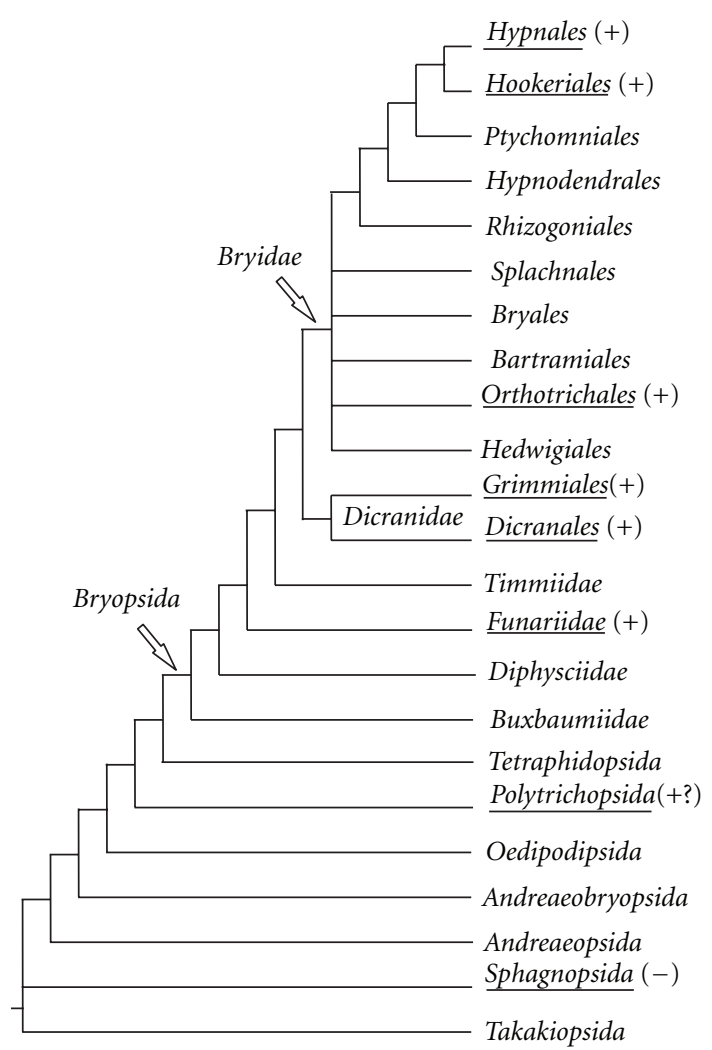

Figure 4: Synoptic consensus view on phylogeny of major Bryophyta taxa based on recent molecular analyses, adopted from Figure 3 of Shaw et al. [26] with modifications. Taxa discussed in the current study are underlined. Taxa from Hypnales (upper) to Dicranales (lower) represent orders; taxa from Timmiidae (upper) to Buxbaumiidae (lower) represent subclasses; taxa from Tetraphidopsida (upper) to Takakiopsida (lower) represent classes. (+) indicates the encoding of miR390-guided TAS3-like genes, and (-) indicates the possible absence of miR390-guided TAS3-like genes. Arrows indicate branching points showing monophyletic origin of class Bryopsida and subclass Bryidae.

$P$. patens loci TAS3a and TAS3d encode rather closely related TAS genes (Figure 1) [15]. However, we found that TAS3d family is unique among TAS genes because it overlaps a protein-coding gene (“GeneID: 5915198"). TAS3d precursor RNA is complementary to the $5^{\prime}$-terminal region of the mRNA (NCBI accession number XM_001751798) and one intron. The encoded protein of unknown function (NCBI accession number XP_001751850) is 292 amino acids in length and has no closely related sequences in other plants. Inspection of nucleotide sequences of $P$. patens TAS3a and related loci described in this paper (Brachythecium latifolium clone 50-Br, Orthotrichum pumilum clone 24-Opu, and Pylaisia polyantha clone 11-Pyp) (Table 1) at Expasy web service (see Section 2) showed that complementary sequences of these four loci cannot encode protein because of the lack of initiation AUG codons or the presence of multiple stop codons although all of them include sequences corresponding to the intron of $P$. patens TAS3d-overlapping protein-coding gene 5915198 (data not shown). Despite this difference, sequence alignment shows obvious similarity between all five TAS precursor genes shown in Figure 2. Most conserved area represents the region including AP2-specific and ARF-specific ta-siRNA sequence blocks and the spacer region between them (Figure 2). It was experimentally shown that this spacer also codes for ta-siRNA in P. patens [23]. Our BLAST analysis at NCBI showed that both complementary sequences of the AP2-ARF spacer can target $P$. patens mRNAs coding for uncharacterized proteins XP_001780327 (186 amino acids in length) and XP_001780322 (382 amino acids in length). There are 3.5 mismatches (counting G: $\mathrm{U}$ wobbles as 0.5 mismatches) between these tasiRNAs from TAS3d locus and each of the targeted mRNAs, which is just at the cutoff for confident computational target prediction [28]. The sequences from Brachythecium latifolium clone 50-Br, Orthotrichum pumilum clone 24-Opu, and Pylaisia polyantha clone 11-Pyp show 6-8 mismatches that can reflect significant evolutionary distance (Figure 4) between these moss species and $P$. patens.

Analysis of the sequence alignments shown in Figures 1 and 2 suggests that only representatives of subclass Bryidae encode TAS3-like genes belonging to P. patens TAS3a and TAS3d families (Table 1). On the other hand, only the Dicranidae species code for the genes closely related to $P$. patens TAS3c family (Figure 2 and Table 1). To support this observation as a conclusive general view on peculiarities of TAS loci evolution in mosses, more detailed analysis of TAS gene diversity is required. Importantly, the activity in this direction can be inferred from the previously published data which strongly suggested the presence of miR390 species and, respectively, TAS3-like RNA precursors in the earlier diverged moss class Polytrichopsida (Figure 4) [24].

Unlike mosses of class Bryopsida showing at least one visible DNA band after PCR amplification, genomic DNAs of the Sphagnopsida mosses from the earliest diverged (except Takakia) Bryophyta lineage (Figure 4), namely, Sphagnum squarrosum and Sphagnum girgensohnii, showed no PCR products with $P$. patens-specific (Figure 1) and dicotspecific miR390-mimicking primers [15] (data not shown). The same negative result was obtained for a lycopod Selaginella kraussiana (Figure 1) that is in accordance with the data reported previously for Selaginella uncinata [24]. This finding was intriguing due to the fact that at least some miRNA families including miR390 have remained essentially unchanged since the last common ancestor of mosses $(P$. patens) and flowering plants $[8,17,24]$. However, during the preparation of the paper, we learned that the completely sequenced genome of Selaginella moellendorffii belonging to the earliest extant tracheophyta lineage having common ancestors with mosses contains no DCL4, RDR6, and MIR390 loci required for the biogenesis of ta-siRNAs [29]. Their absence suggests that ta-siRNA-dependent processes in plants are regulated differently in distinct plant lineages and possibly reflects the independent evolutionary history (retention or loss) of the TAS genes in separate taxons. Moreover, our results support that purifying selection is one of the main evolutionary forces acting on TAS3-like genes, maintaining lower levels of sequence divergence in mature ta-siRNAs (AP2-specific and ARF-specific sequences of precursors) than in their flanking regions (Figures 2 and 3). Although we 
identified several mutations in the functional regions of the candidate ta-siRNA genes in mosses, such as in ta-siARF, tasiAP2, and the miR390 binding sites (Figures 2 and 3), it does not necessarily indicate a direct consequence of the positive selection events. Further experiments involving identification of resulting phenotypic changes are needed to reveal and prove the events of positive selection.

\section{Acknowledgments}

The authors thank M. S. Ignatov (N. V. Cycin Main Botanical Garden RAS) for providing moss specimens and their identification and M. M. Pooggin (Institute of Botany, University of Basel) for stimulating discussions. This work was supported by Grants 09-04-01324-a and 09-04-00707-a from the Russian Foundation for Basic Research.

\section{References}

[1] X. Chen, "Small RNAs - secrets and surprises of the genome," Plant Journal, vol. 61, no. 6, pp. 941-958, 2010.

[2] M. J. Axtell, J. O. Westholm, and E. C. Lai, "Vive la différence: biogenesis and evolution of microRNAs in plants and animals," Genome Biology, vol. 12, no. 4, pp. 221-234, 2011.

[3] S. Röther and G. Meister, "Small RNAs derived from longer non-coding RNAs," Biochimie, vol. 93, no. 11, pp. 1905-1915, 2011.

[4] N. R. Gursanscky, I. R. Searle, and B. J. Carroll, "Mobile microRNAs hit the target," Traffic, vol. 12, no. 11, pp. 14751482, 2011.

[5] E. Bonnet, Y. van de Peer, and P. Rouzé, “The small RNA world of plants," New Phytologist, vol. 171, no. 3, pp. 451-468, 2006.

[6] Z. Xie and X. Qi, "Diverse small RNA-directed silencing pathways in plants," Biochimica et Biophysica Acta, vol. 1779, no. 11, pp. 720-724, 2008.

[7] X. Chen, "Small RNAs and their roles in plant development," Annual Review of Cell and Developmental Biology, vol. 25, pp. 21-44, 2009.

[8] C. Lelandais-Brière, C. Sorin, M. Declerck, A. Benslimane, M. Crespi, and C. Hartmann, "Small RNA diversity in plants and its impact in development," Current Genomics, vol. 11, no. 1, pp. 14-23, 2010.

[9] X. Chen, "MicroRNA metabolism in plants," Current Topics in Microbiology and Immunology, vol. 320, pp. 117-136, 2008.

[10] H. Zhang and J.-K. Zhu, "RNA-directed DNA methylation," Current Opinion in Plant Biology, vol. 14, no. 2, pp. 142-147, 2011.

[11] S. W. L. Chan, "Inputs and outputs for chromatin-targeted RNAi," Trends in Plant Science, vol. 13, no. 7, pp. 383-389, 2008.

[12] M. J. Axtell and J. L. Bowman, "Evolution of plant microRNAs and their targets," Trends in Plant Science, vol. 13, no. 7, pp. 343-349, 2008.

[13] A. C. Mallory and N. Bouché, "MicroRNA-directed regulation: to cleave or not to cleave," Trends in Plant Science, vol. 13, no. 7, pp. 359-367, 2008.

[14] B. Merget and M. Wolf, "A molecular phylogeny of Hypnales (Bryophyta) inferred from ITS2 sequence-structure data," BMC Research Notes, vol. 3, pp. 320-327, 2010.

[15] M. S. Krasnikova, I. A. Milyutina, V. K. Bobrova et al., "Novel miR390-dependent transacting siRNA precursors in plants revealed by a PCR-based experimental approach and database analysis," Journal of Biomedicine and Biotechnology, vol. 2009, Article ID 952304, 9 pages, 2009.

[16] H. Vaucheret, "MicroRNA-dependent trans-acting siRNA production," Science's STKE, vol. 2005, no. 300, p. pe43, 2005.

[17] E. Allen and M. D. Howell, "miRNAs in the biogenesis of trans-acting siRNAs in higher plants," Seminars in Cell and Developmental Biology, vol. 21, no. 8, pp. 798-804, 2010.

[18] A. Takeda, S. Iwasaki, T. Watanabe, M. Utsumi, and Y. Watanabe, "The mechanism selecting the guide strand from small RNA duplexes is different among argonaute proteins," Plant and Cell Physiology, vol. 49, no. 4, pp. 493-500, 2008.

[19] E. Allen, Z. Xie, A. M. Gustafson, and J. C. Carrington, "microRNA-directed phasing during trans-acting siRNA biogenesis in plants," Cell, vol. 121, no. 2, pp. 207-221, 2005.

[20] M. J. Axtell, C. Jan, R. Rajagopalan, and D. P. Bartel, "A twohit trigger for siRNA biogenesis in plants," Cell, vol. 127, no. 3, pp. 565-577, 2006.

[21] H. M. Chen, Y. H. Li, and S. H. Wu, "Bioinformatic prediction and experimental validation of a microRNA-directed tandem trans-acting siRNA cascade in Arabidopsis," Proceedings of the National Academy of Sciences of the United States of America, vol. 104, no. 9, pp. 3318-3323, 2007.

[22] M. J. Axtell, J. A. Snyder, and D. P. Bartel, "Common functions for diverse small RNAs of land plants," Plant Cell, vol. 19, no. 6, pp. 1750-1769, 2007.

[23] M. Talmor-Neiman, R. Stav, L. Klipcan, K. Buxdorf, D. C. Baulcombe, and T. Arazi, "Identification of trans-acting siRNAs in moss and an RNA-dependent RNA polymerase required for their biogenesis," Plant Journal, vol. 48, no. 4, pp. 511-521, 2006.

[24] M. J. Axtell and D. P. Bartel, "Antiquity of microRNAs and their targets in land plants," Plant Cell, vol. 17, no. 6, pp. 16581673, 2005.

[25] T. Zhao, G. Li, S. Mi et al., "A complex system of small RNAs in the unicellular green alga Chlamydomonas reinhardtii," Genes and Development, vol. 21, no. 10, pp. 1191-1203, 2007.

[26] A. J. Shaw, P. Szövényi, and B. Shaw, "Bryophyte diversity and evolution: windows into the early evolution of land plants," American Journal of Botany, vol. 98, no. 3, pp. 352-369, 2011.

[27] A. Li and L. Mao, "Evolution of plant microRNA gene families," Cell Research, vol. 17, no. 3, pp. 212-218, 2007.

[28] M. W. Jones-Rhoades, D. P. Bartel, and B. Bartel, "MicroRNAs and their regulatory roles in plants," Annual Review of Plant Biology, vol. 57, pp. 19-53, 2006.

[29] J. A. Banks, T. Nishiyama, M. Hasebe et al., "The selaginella genome identifies genetic changes associated with the evolution of vascular plants," Science, vol. 332, no. 6032, pp. 960963, 2011. 


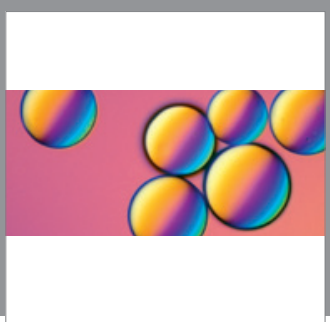

Peptides

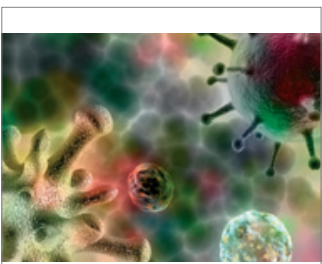

\section{Virology}

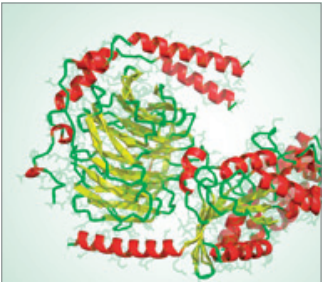

Signal Transduction
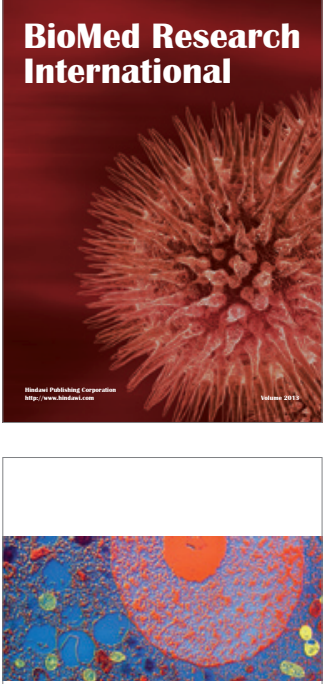

ISRN

Cell Biology
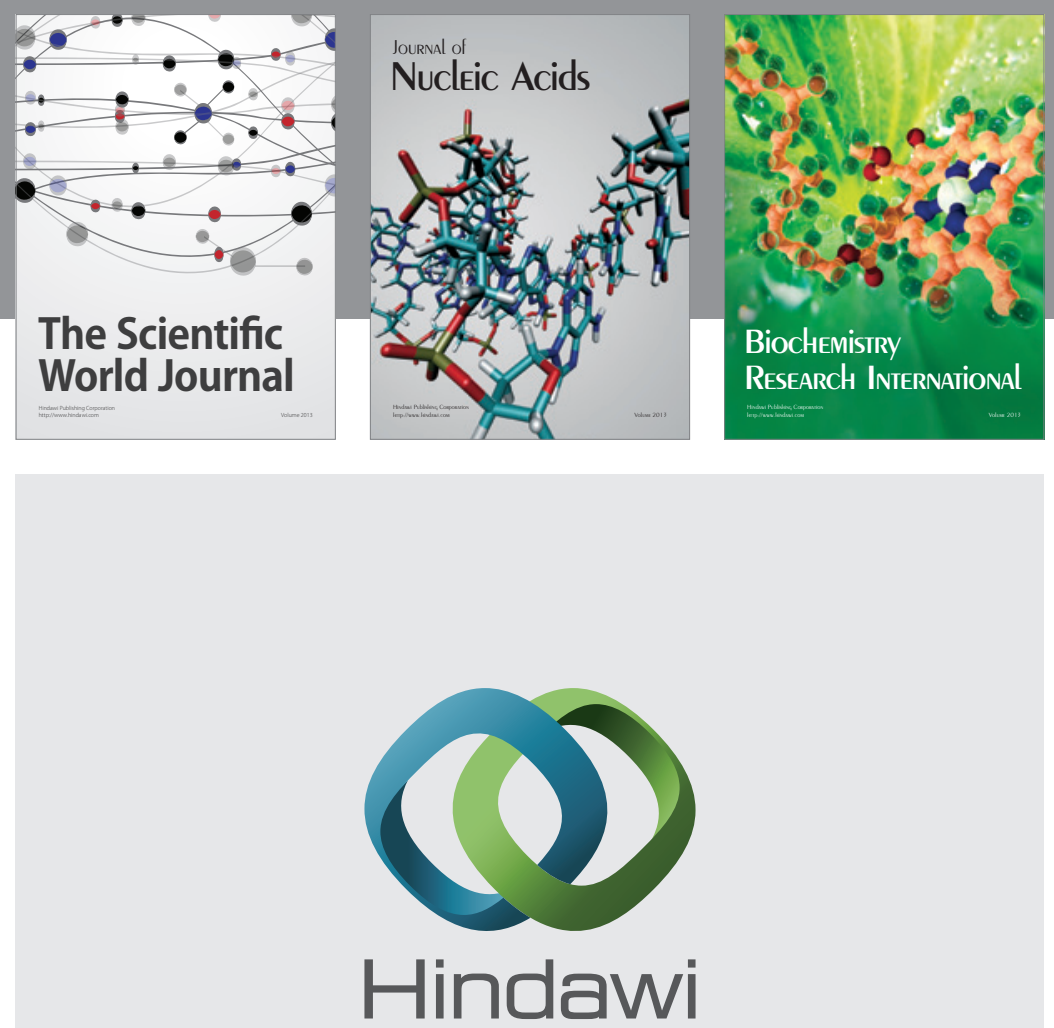

Submit your manuscripts at http://www.hindawi.com

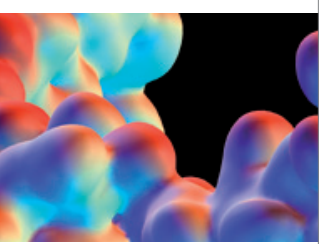

Enzyme Research

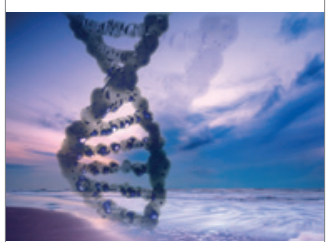

Evolutionary Biology
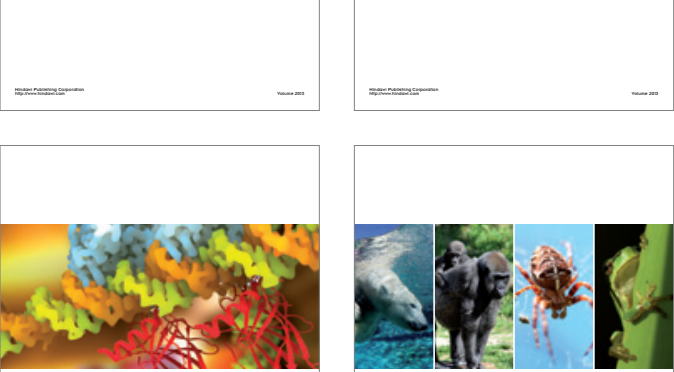

ISRN

Molecular Biology

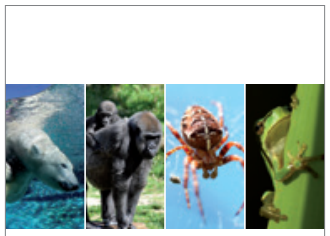

ISRN Zoology

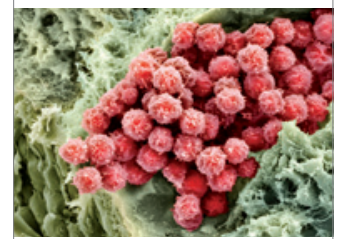

Stem Cells International

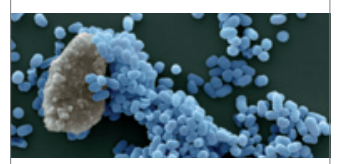

ISRN

Biotechnology
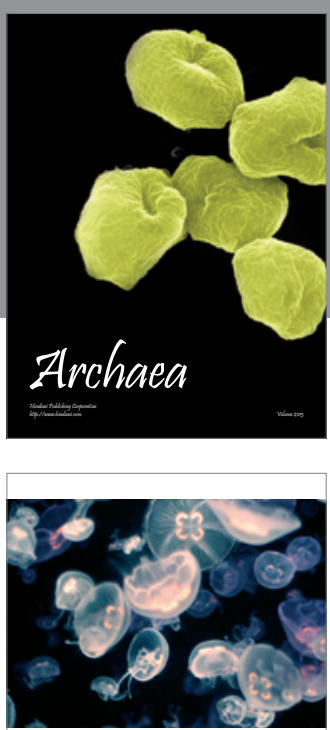

Journal of Marine Biology
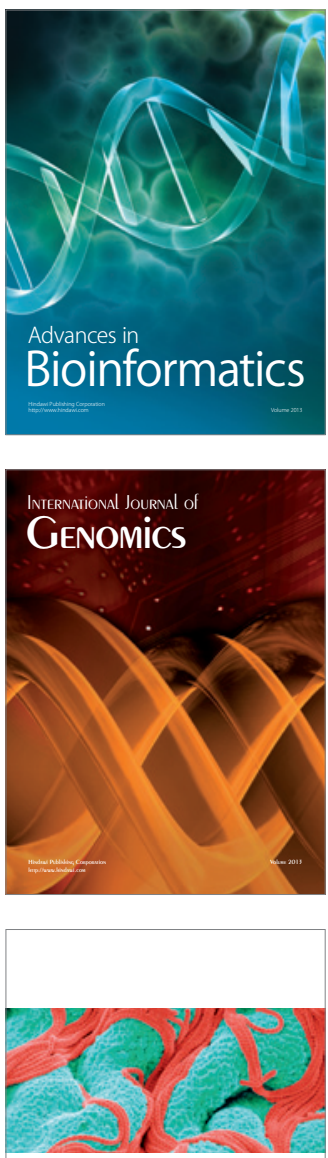

ISRN

Microbiology 